\title{
Lubrication performance analysis of sealing structures in rodless open cylinders
}

\author{
JIANLIN ZHONG ${ }^{1} \mathbb{D}$, DAWEI MA ${ }^{1}$, JIE REN $^{1, *}$ and LIN YAO ${ }^{2}$ \\ ${ }^{1}$ School of Mechanical Engineering, Nanjing University of Science and Technology, Nanjing 210094, China \\ ${ }^{2}$ Sixtieth Institute of the General Staff, Nanjing 210016, China \\ e-mail: zhongjianlin@njust.edu.cn
}

MS received 25 March 2018; revised 17 December 2018; accepted 23 January 2019; published online 27 March 2019

\begin{abstract}
As the lubrication performance of sealing structures in rodless open cylinders varies with sliding velocity, the concept of a critical velocity is proposed based on the aforementioned characteristics and the lubrication performance of sealing structure is analysed. A finite-element model of sealing structure is established and the oil film load under dynamic lubrication condition is obtained. A two-dimensional theoretical model of lubrication performance in sealing structure is established based on the proposed critical velocity. The influence on critical velocity of parameters including pre-compression, geometry size and microscopic morphology is analysed. Considering surface microscopic morphology, generation of heat by friction, heat flux distribution and other factors, a three-dimensional numerical model of lubrication performance of sealing structure is established to reveal the influence of texture mechanism on lubrication performance. As concluded, with the increase of pre-compression, the lip angle and the temperature of lubrication oil, the critical velocity increases. The three-dimensional microscopic morphology of seal ring is found to pose obvious influence on critical velocity, and the isotropic microscopic morphology tends to form total lubrication under the same conditions.
\end{abstract}

Keywords. Rodless open cylinder; sliding seal; lubrication performance; critical velocity; microscopic morphology.

\section{Introduction}

Rodless open cylinders are a type of pneumatic actuators for special utilities and composed of an open actuator, transmission piston, seal and other components. A linear drive with high velocity for massive objects can be achieved using rodless open cylinders, which owns the features of high velocity, long stroke and large load. Rodless open cylinders can be widely applied in the fields of industrial automation, aerospace, ship and marine as well as weaponry equipment $[1,2]$. For a certain type of rodless open cylinder, its velocity could increase to $30 \mathrm{~m} / \mathrm{s}$ within $0.5 \mathrm{~s}$ and the average pressure of working medium could reach $20 \mathrm{MPa}$. The temperature vibration rate in the cylinder can be $200^{\circ} \mathrm{C} / \mathrm{s}$ and the working temperature ranges from -25 to $50^{\circ} \mathrm{C}$, which exhibits obvious characteristics of large-span variation of $P V$ value. The extreme working environment causes the sealing lubrication problem to become one of the bottlenecks that restrict the performance improvement of the actuator.

In view of studies on lubrication performance of highvelocity linear sliding seal, Rana and Sayles [3] have

*For correspondence conducted experiments to study the friction characteristics of elastic reciprocating sealing ring of aviation hydraulic actuator, which shows that the maximum friction forces occur at the start and the stop of the actuator. Bhaumik et al [4] designed an improved experimental device for studying contact mechanical characteristics of reciprocating hydraulic seals. Nikas et al [5] studied the influence laws of different surface roughness and reciprocating sliding velocities on leakage and frictional characteristics. In accordance with these research conclusions, significant value of reference has been achieved for the design and numerical analysis of the lubrication state of the seal ring. However, it is difficult to obtain the oil film characteristics of hydrodynamic lubrication film directly through experimental tests with limited test conditions, and the prediction and optimization of the sealing performance can hardly be achieved. Thus, numerical simulation method is proposed to be an effective method. Salant et al [6] established the two-dimensional elastohydrodynamic lubrication (EHL) model of reciprocating seal ring for hydraulic actuator. The distribution of contact pressure of pre-compression under the action of fluid pressure is compared to the distribution of dynamic contact pressure. Then, with the transient EHL model of hydraulic actuator, Thatte and Salant [7] studied 
the medium pressure, velocity of piston rod and internal/ external stroke, both of which have influences on hydrodynamic lubrication characteristics. Based on the assumption of mixed lubrication, Yang and Salant [8] and Yang et al [9] established the fluid-solid coupling seal lubrication models in two-dimensional plane for O-shaped ring and U-shaped ring as well as double-lip U-shaped ring and HBTS in the hydraulic actuator. The U-shaped reciprocating seal ring with "double-lip" structure is proposed and the seal performance for different actuator velocities is analysed. The results show that the double-lip U-shaped ring owns the optimum sealing effect. Considering the strong coupling between elastic deformation equation of reciprocating seal and hydrodynamic lubrication equation (Reynolds equation), Schmidt et al [10] put forward a transient two-dimensional finite-element method with the introduction of flow factor, which is equivalent to the surface roughness. Also, EHL characteristics of rectangular reciprocating seals have been analysed. Regarding the influence of surface roughness, Crudu et al [11] established a numerical simulation model of a reciprocating sliding seal ring, namely a "U-shaped ring". The calculation of friction force has been simulated and compared to test results. The result indicates that both of them match quite well. Huang [12] and Huang and Salant [13, 14] adopted the coupling method of fluid dynamics and elasticity to obtain the distribution of contact pressure, which is equivalent to the oil film pressure. The balance point between oil film load and dynamic contact load of seal ring was sought by reverse solution of Reynolds equation. Later, the seal and lubrication performances of reciprocating piston rod were studied for the conditions of abundant and deficient lubrication oil.

In the light of existing literature, the research works on linear sliding seal lubrication are generally based on the assumption of mixed lubrication or complete lubrication; there are hardly any research works on the transformation process from the mixed lubrication to complete lubrication. In addition, as most studies were based on two-dimensional seal lubrication model, the influences of three-dimensional microscopic morphology in seal ring on film characteristics and lubrication states could not be analysed by the current study.

The proposed rodless open cylinder adopts the VL sealing structure with local structural improvement to enhance its hydrodynamic effect. Firstly, based on the idea that the normal load is completely taken up by the oil film, a finite-element model of the sealing structure is established to obtain the load of oil film in the dynamic lubrication state. Later, the concept of critical velocity is proposed and a two-dimensional model of lubrication performance of sealing structure is established. In the full range of velocity, preliminary positioning on "critical velocity" has been carried out to reveal the influence mechanism of pre-compression, lip angle and temperature on the lubrication performance. Finally, an AR (auto-regression) model of digital filtering technology is adopted to construct three-dimensional microscopic domain of oil film. Considering the principle of heat generation by friction and heat flow distribution, a three-dimensional accurate analysis model of the lubrication state of the seal ring is established. Furthermore, the influence mechanism of texture feature on lubrication is revealed by precise positioning of critical velocity. In conclusion, the research methods and results can support the design of seal lubrication and prediction of evolution of lubrication state for rodless open cylinders.

\section{Sealing performance analysis of sealing structure}

\subsection{Sealing design of ejection device}

As shown in figure 1, the rodless open cylinder mainly consists of a gas source, pneumatic valve, open cylinder, piston, loading bracket and the sealing structure. When the pneumatic valve is opened, the high-pressure working substance in the gas source enters the opening cylinder and drives the piston and the loading bracket to move, thus realizing the high-speed linear driving of loading. The VL sealing structure used in the open cylinder researched in this paper is composed of a $\mathrm{V}$-shaped ring and an $\mathrm{O}$-shaped ring [15]; improvement on lip surface of $\mathrm{V}$-shaped ring has been carried out as eliminating the lip angle near contact domain and enlarging the lip angle of other domain, which could enhance the fluid dynamic pressure effect of lip angle as well as avoid the phenomenon of oil lack on sliding contact surface.

\subsection{Finite-element model of sealing structure}

Nitrile rubber (NBR) is adopted as the material of O-shaped ring for resilient element, and hardness-enhanced wear-resistant polytetrafluoroethylene (PTFE) is used as the material of $\mathrm{V}$-shaped ring for sliding seal contact element. The V-shaped ring is in direct contact with the inner wall surfaces of cylinder and the seal steel strip, and the contact surface is the sliding seal contact surface. The mechanical properties of NBR and PTFE are, respectively, described by a two-parameter M-R hyper-elastic model and linear elastic model. It can be concluded according to the static compressive test that the hardness-enhanced wear-resistant PTFE is approximately linear elastic within the range of small deformation, and the elastic modulus is about $350 \mathrm{MPa}$.

The two-parameter M-R model is

$$
U=C_{10}\left(\bar{I}_{1}-3\right)-C_{01}\left(\bar{I}_{2}-3\right)+D\left(J^{e l}-1\right)^{2}
$$

where $U$ is strain energy, $\bar{I}_{1}$ and $\bar{I}_{2}$ are partial strain ratios, $J^{e l}$ is volume rate and $C_{10}, C_{01}, D$ are relative coefficients. Relevant parameters are as follows. 


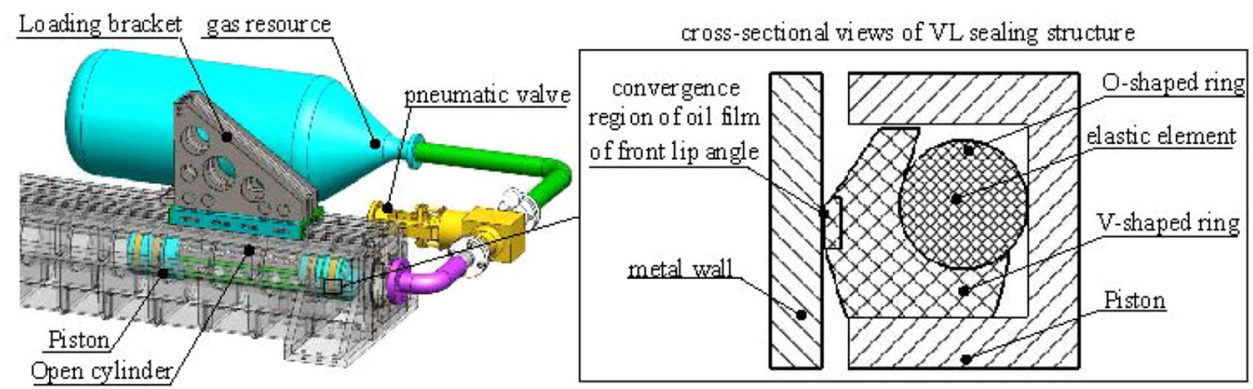

Figure 1. Sealing structure.

(1) NBR: $C_{10}=0.202, C_{01}=6.858$ and $D=0$;

(2) PTFE: $E=350 \mathrm{MPa}$ and the Poisson ratio is $v=0.4$.

The VL seal macroscopic contact model is established in the nonlinear finite-element software; contact pairs in the model mainly include the O-shaped ring and mounting groove, $\mathrm{O}$-shaped ring and $\mathrm{V}$-shaped ring, $\mathrm{V}$-shaped ring and mounting groove as well as $\mathrm{V}$-shaped and wall surface. The contact constraint adopts the method of penalty function, and the contact search algorithm adopts master-subordinate global search algorithm. Also, the mesh density of contact domain is increased as shown in figure 2; the type of contact elements is CPS4, the number of contact elements is 1648 and the master and slave surface are highlighted in red and purple.

\subsection{Seal performance analysis of sealing structure}

The load application is divided into two steps: (1) applying different pre-compressions, the pre-compression is converted to the displacement of the metal wall, and the metal wall is set to move inward for a period of displacement in the finite-element model; (2) applying medium pressure of $20 \mathrm{MPa}$, which is according to the normal working pressure of the rodless open cylinder. The contact pressure nephograms of VL seal ring with medium pressure of 0 and 20 $\mathrm{MPa}$ are shown in figure 3 , where the lip angle is $10^{\circ}$ and the pre-compression is $16 \%$ [16]. As indicated, when the

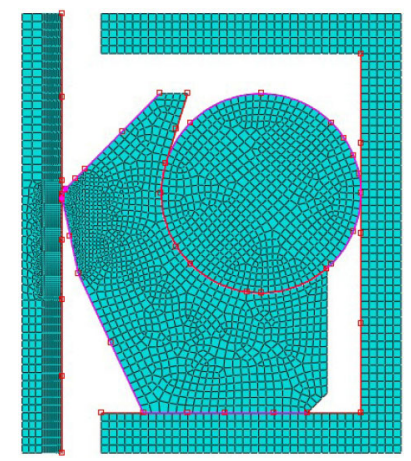

Figure 2. Mesh model of VL seal ring. medium pressure is $0 \mathrm{MPa}$, the $\mathrm{VL}$ seal is under the installing condition with better coordination between $\mathrm{O}$-shaped ring and $\mathrm{V}$-shaped ring. The maximum contact pressure between $\mathrm{V}$-shaped ring and actuator rod reaches $100 \mathrm{MPa}$, where the fluid could be well sealed. After the pressure of seal medium reaches $20 \mathrm{MPa}$, the maximum contact pressure will increase to a certain value, which will be far larger than the pressure of seal medium; thus the seal requirement can be satisfied.

\section{Two-dimensional high-efficiency theoretical analysis model for lubrication performance}

\subsection{The concept of critical velocity}

Whether the two surfaces are completely separated by oil film or partially contacted with micro-convex bodies depends on the base thickness of oil film $h$ and the comprehensive roughness $\sigma$ of two surfaces [17]. The lubrication condition is generally determined by the film thickness ratio $\lambda$ and three main lubrication conditions are as follows.

(1) Hydrodynamic lubrication: the friction surfaces are totally isolated and the film thickness ratios are 3-5.

(2) Mixed lubrication: the contact load is assumed by the oil film and the asperity jointly and the film thickness ratios are 1-3.

(3) Boundary lubrication: as the effect of hydrodynamic lubrication is very small, the contact loads are almost all assumed by asperity, and the film thickness ratio $\lambda$ is smaller than 1 .

Based on the distribution method of normal contact load, it is considered that the load assumed by asperity is zero while the total seal contact load is assumed by oil film during the transform of mixed lubrication to hydrodynamic lubrication [18]. Therefore, the total load of oil film in contact domain equals the total contact load.

In line with the basis mentioned in previous paragraph, the concept of "critical velocity" is proposed as follows. With the increasing of relative sliding velocity of contact friction pairs form zero gradually, when the film thickness ratio $\lambda$ of pressure oil film reaches 3 and the total load of oil 


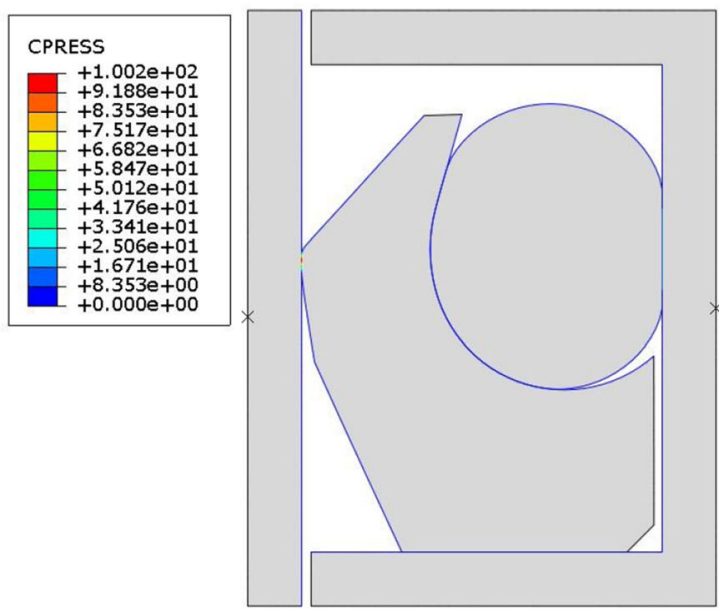

(a) Pre-compression of 16\% with medium pressure of $0 \mathrm{MPa}$
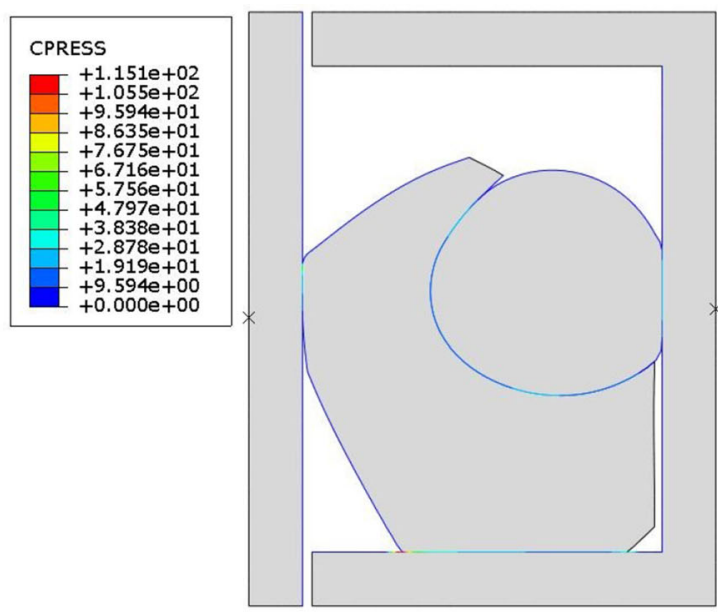

(b) Pre-compression of $16 \%$ with medium pressure of $20 \mathrm{MPa}$

Figure 3. Stress nephogram of VL seal contact.

film in contact domain equals the total load, the sliding velocity at this time is known as "critical velocity" and the condition is the critical condition, as shown in figure 4. The "critical velocity" is the key index that determines the transformation condition of seal sliding contact surface from mixed lubrication to hydrodynamic lubrication. The lubrication characteristics of the sealing structure in the rodless open cylinder are then researched in this paper. In order to simplify the problem, the rough surface of the seal ring is established by the comprehensive roughness method, and the metal wall is equivalent to the smooth wall surface.

\subsection{Establishment of two-dimensional efficient analysis model}

The lubrication problem of the sealing structure could be simplified as lubrication problem of an infinitely wide slider without considering end leakage. The generalized threedimensional Reynolds equation could be reduced to onedimensional Reynolds equation in the form $[19,25]$

$$
\frac{\mathrm{d}}{\mathrm{d} x}\left(\frac{h^{3}}{\eta} \frac{\mathrm{d} p}{\mathrm{~d} x}\right)=6 V \frac{\mathrm{d} h}{\mathrm{~d} x} .
$$

The pressure distribution of oil film could be obtained using Eq. (2) by substitution of boundary condition when the film

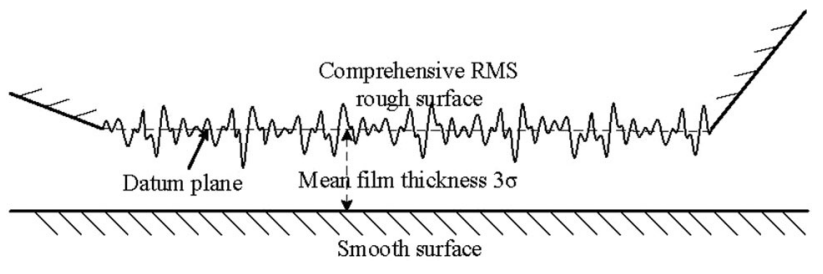

Figure 4. Schematic diagram of critical state. thickness equation is known. For the lubrication problem of sealing structure in this paper, the boundary conditions of pressure entrance and existence are, respectively, the pressure of working medium in cylinder and the air pressure. The film thickness equation could be obtained by the finite-element model.

For programming, set $X=\frac{x}{L}, H=\frac{h}{h_{1}}$ and $P=\frac{p h_{1}^{2}}{6 \eta V L}$, where $h_{1}$ is the film thickness of pressure entrance, $\eta$ is the viscosity of lubrication oil and $\eta=0.1$ at the reference temperature. The dimensionless Reynolds equation has the form

$$
\frac{\mathrm{d}}{\mathrm{d} X}\left(H^{3} \frac{\mathrm{d} P}{\mathrm{~d} X}\right)=\frac{\mathrm{d} H}{\mathrm{~d} X}
$$

The boundary conditions of pressure are $p_{x=-L}=$ $101325 \mathrm{~Pa}$ and $p_{x=0}=20265000 \mathrm{~Pa}$. The dimensionless pressure boundary condition can be obtained as $P=\frac{p h_{1}^{2}}{6 \eta V L}$.

The relation between $H$ and $X$ can be obtained as follows:

(1) In the macroscopic finite nonlinear contact model, measuring the distances from each node on the contact surface to the rigid plane.

(2) Based on the $3 \sigma$ division criterion of hydrodynamic lubrication condition, the measured distances from each node to rigid plane are offset by $3 \sigma$ positively.

(3) The endpoint of convergence angle corresponding to the position of rigid plane is set as the origin of coordinates. Later, the coordinate of each node relative to the origin can be obtained according to the distance from each offset node to rigid plane.

(4) The height equation from the basic contact plane of seal ring to rigid plane, namely the relation between film thickness $h$ and contact coordinate, can be obtained by least-square fitting. 
(5) The relation between $H$ and $X$ could be obtained by dimension normalization.

The pressure expression of oil film is [20]

$$
P(i)=\frac{H_{i+1 / 2}^{3} P_{i+1}+H_{i-1 / 2}^{3} P_{i-1}+\Delta X\left(H_{i+1 / 2}-H_{i-1 / 2}\right)}{H_{i+1 / 2}^{3}+H_{i-1 / 2}^{3}} .
$$

The difference method is used to discretize Eq. (4) without considering the variations of pressure in oil film along thickness. Based on the output node coordinate $X(i)$, node pressure $P(i)$ and node film thickness $H(i)$, the actual film thickness of oil film and actual pressure distribution can be obtained.

\subsection{Preliminary positioning of critical velocity}

The numerical solution steps of preliminary positioning for critical velocity are as follows:

(1) According to macroscopic analysis, the film thickness equation can be fitted.

(2) The relation between $H$ and $X$ can be obtained by dimension normalization.

(3) The calculation domain along $X$ direction can be discretized as 1001 points, i.e., the dimensionless film thickness $H(i)$ corresponding to node $X(i)$ can be obtained.

(4) The dimensionless pressure boundary can be obtained by combining pressure boundary as well as the given preliminary wall velocity $V$.

(5) Combined with oil film pressure (Eq. (4)), the oil film pressure of each node in calculation domain can be calculated with the given preliminary pressure of oil film.

(6) In order to guarantee the convergence, the relaxation iteration method is adopted to correct the node pressure, i.e. $P_{i}^{k+1}=(1-\alpha) P_{i}^{k}+\alpha \hat{P}_{i}^{k}$, where $P_{i}^{k+1}$ is the update node pressure, $P_{i}^{k}$ is the current node pressure, $\hat{P}_{i}^{k}$ is the pressure obtained from Eq. (4) and $\alpha$ is a positive number less than 1 , which is set as 0.7 in this paper.

(7) The criterion of relative precision is used as an iterative convergence condition, and the convergence precision is 0.0001 , i.e.

$$
\frac{\sum_{I=1}^{N}\left|p_{i}^{k}-p_{i}^{k-1}\right|}{\sum_{I=1}^{N} p_{i}} \leq 0.0001 .
$$

(8) The output node pressure is used to determine whether the critical condition is reached; if yes, then the initial positioning value of critical velocity is the output. Otherwise, the initial velocity of wall surface is changed and the afore-mentioned steps are repeated until the critical conditions are satisfied. The flowchart of solution for initial positioning of critical velocity is shown in figure 5 .

The critical velocity and the maximum pressure of oil pressure of three design parameters including different precompressions, different lip angles and different comprehensive RMS roughness values $\sigma$ are obtained at the lubrication oil temperature of $25^{\circ} \mathrm{C}$, as shown in table 1 .

\subsection{Influence of different factors on lubrication performance}

The radial basis neural network method is used to train the obtained sample data to obtain an approximate model of critical velocity of the seal ring. Sensitivity analysis of the influence factors on critical velocity was carried out by combining the multivariate quadratic regression method and the optimized Latin hypercube experimental design method [21]. The sensitivity analysis results of influence factors are presented in figure 6 . As indicated, the critical velocity has the largest influence on the sensitivity of

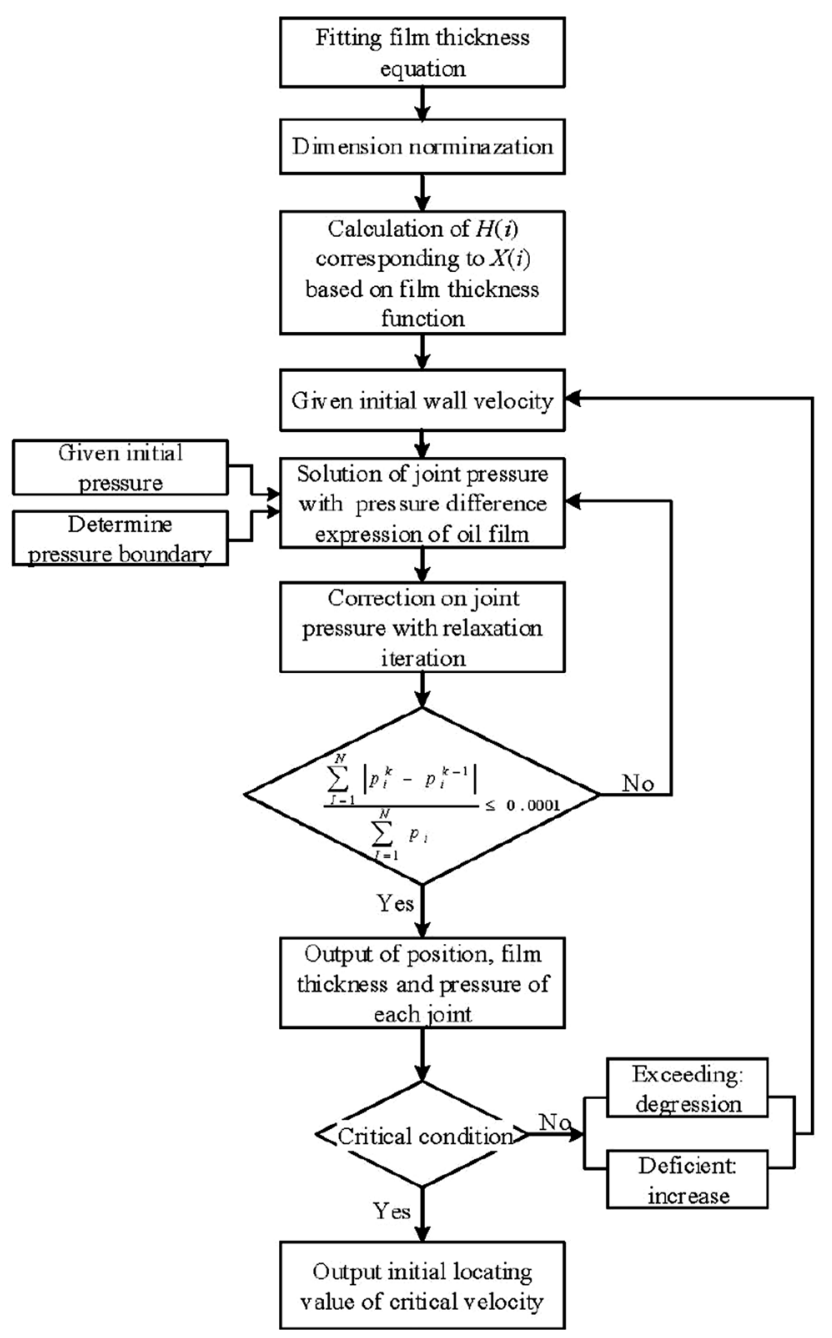

Figure 5. Flowchart of critical velocity calculation. 
Table 1. Sample space.

\begin{tabular}{lccccc}
\hline $\begin{array}{l}\text { Sample } \\
\text { number }\end{array}$ & $\begin{array}{c}\text { Pre-compression } \\
(\%)\end{array}$ & $\begin{array}{c}\text { Lip angle } \\
(\mathrm{deg})\end{array}$ & $\begin{array}{c}\text { Roughness } \\
(\mu \mathrm{m})\end{array}$ & $\begin{array}{c}\text { Critical velocity } \\
(\mathrm{m} / \mathrm{s})\end{array}$ & $\begin{array}{c}\text { Maximum pressure of oil film } \\
(\mathrm{MPa})\end{array}$ \\
\hline 1 & 14 & 8 & 0.6 & 4.8 & 13.3 \\
2 & 14 & 8 & 1 & 8.6 & 12.6 \\
3 & 14 & 8 & 1.4 & 11 & 17.3 \\
4 & 16 & 8 & 0.6 & 5.5 & 16.1 \\
5 & 16 & 8 & 1 & 10 & 15.2 \\
6 & 16 & 8 & 1.4 & 14 & 26.5 \\
$\ldots$ & 16 & 12 & 1.4 & 20 & 32.35 \\
30 & 18 & 12 & 0.6 & 7.2 & 31.2 \\
31 & 18 & 12 & 1 & 19.2 & 25 \\
32 & 18 & 12 & 1.4 & 26.7 & 36.5 \\
33 & 20 & 12 & 0.6 & 6.5 & 34 \\
34 & 20 & 12 & 1 & 24 & 32.5 \\
35 & 20 & 12 & 1.4 & & \\
36 & 18 & &
\end{tabular}

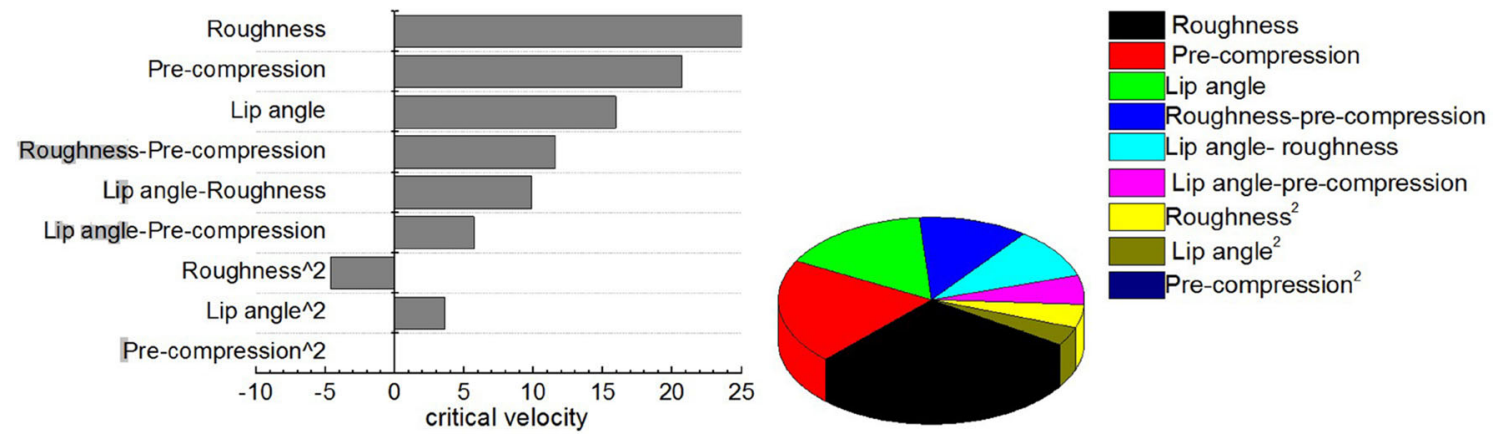

Figure 6. Sensibility analysis with Pareto.

roughness. Meanwhile, the influences of roughness, precompression and lip angle on critical velocity all exhibit positive effect. In addition, the sensitivity of interaction effect is higher, while that of second-order main effect is lower and can be ignored.

The smaller the comprehensive RMS roughness $\sigma$, the lower the critical velocity. In order to reduce the critical velocity, the machining accuracy of seal contact surface should be improved as much as possible. The comprehensive RMS roughness $\sigma$ for seal surface for aviation actuator has reached $0.2 \mu \mathrm{m}$ in literature [15]. It is expensive and difficult to achieve such high machining accuracy for the rodless open cylinder with high-pressure pneumatic ejection device. Therefore, the range of comprehensive RMS roughness $\sigma$ is set as $1 \mu \mathrm{m}$.

In addition, as the temperature has large influence on viscosity of lubrication oil, the maximum pressure of critical velocity and oil film are also obviously influenced by temperature. The temperatures of lubrication oil are $-25,0$, 25 and $50^{\circ} \mathrm{C}$, which correspond to the variation curves of critical velocity as shown in figure 7 . As indicated in figure 7 , the critical velocity is $1.4 \mathrm{~m} / \mathrm{s}$ at $25^{\circ} \mathrm{C}$, which reaches up to $23 \mathrm{~m} / \mathrm{s}$ at $50^{\circ} \mathrm{C}$. Due to the viscosity temperature effect of lubricating oil, the viscosity decreases obviously along with the temperature, and the capacity to form the oil film pressure is markedly reduced. Thus, the higher the temperature of lubrication oil, the higher the critical velocity. However, if the oil temperature is relatively low with a large viscosity, it is difficult to form lubricating oil film due to the poor fluidity. In conclusion, when the

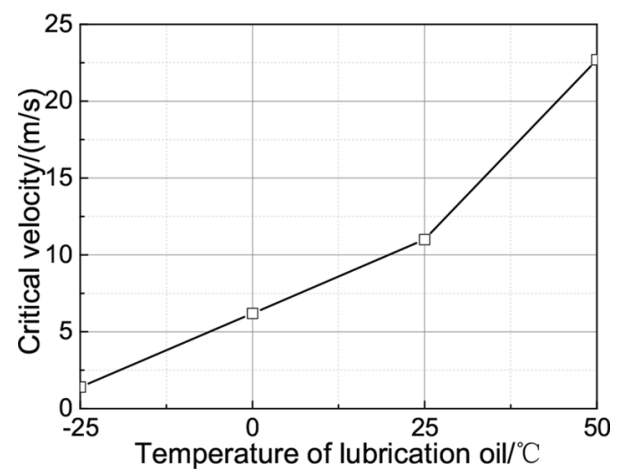

Figure 7. Variation curve of critical velocity. 
rodless open cylinder with high-pressure pneumatic ejection device operates at low temperatures, the low-viscosity lubrication oil at low temperature should be used to form lubrication oil film as soon as possible. For high-temperature operating environment, lubrication oil with large viscosity at high temperature should be selected to improve the load-bearing capacity of oil film.

The initial positioning of critical velocity is achieved with the established two-dimensional high-efficiency analysis mo-del, which provides initial calculation velocity for further precise positioning of the critical velocity. When calculating the precise positioning of critical velocity, the obvious shrinkage of the velocity ranges can improve the calculation efficiency of precise solution on critical velocity in sealing structure. The three-dimensional precise numerical analysis model is based on the selected condition, in which the precompression of seal ring is $16 \%$, the lip angle is $10^{\circ}$, the temperature of lubrication oil is $25^{\circ} \mathrm{C}$ and the comprehensive RMS roughness $\sigma$ is $11 \mu \mathrm{m}$. The critical velocity in this condition is set to $11 \mathrm{~m} / \mathrm{s}$ preliminarily on the basis of twodimensional high-efficiency theoretical analysis model.

\section{Three-dimensional precise numerical model for lubrication performance}

\subsection{Numerical simulation of morphology of sliding contact surface}

The morphology of seal surface is random with Gauss distribution for the height of surface profile. Using the digital filtering technique for AR model, the seal contact rough surface with Gauss distribution feature is generated by computer simulation [22]. Based on design parameters of VL seal ring, the comprehensive surface RMS roughness is set to $1 \mu \mathrm{m}$; the length and width are set, respectively, to

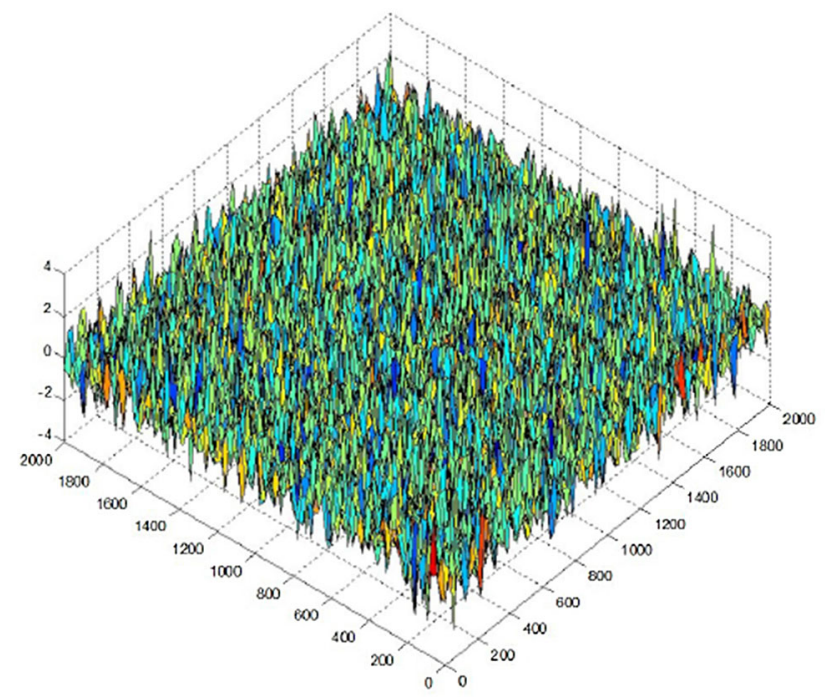

Figure 8. Isotropic texture.

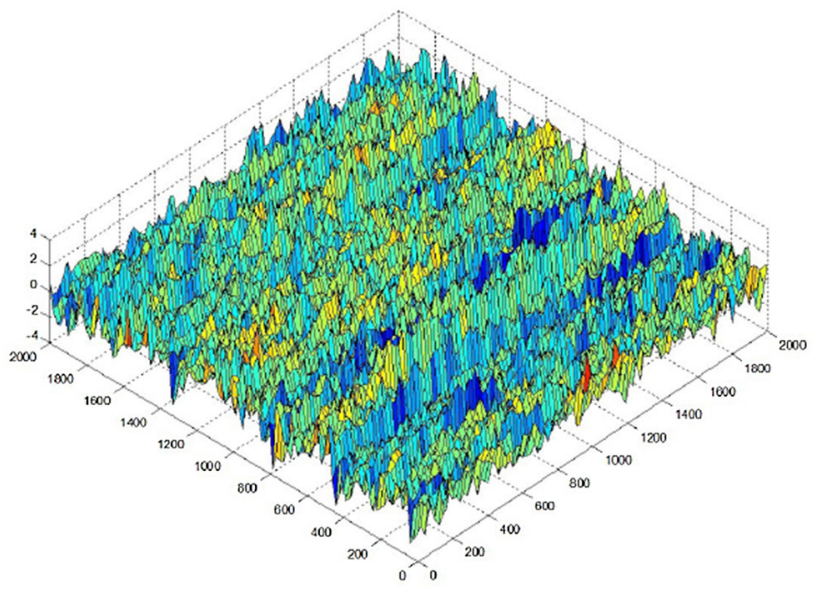

Figure 9. Transverse texture.

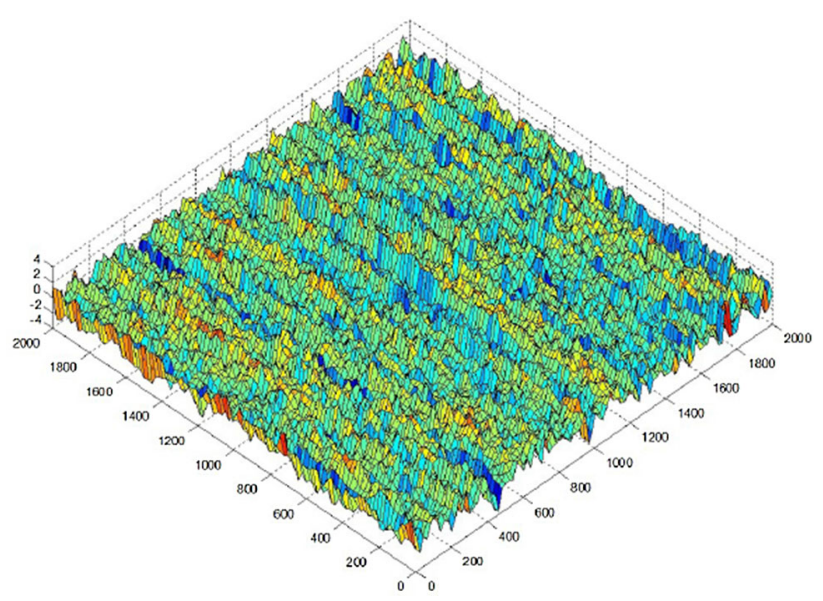

Figure 10. Longitudinal texture.

$2000 \mu \mathrm{m} \times 2000 \mu \mathrm{m}$. Figures 8,9 and 10, respectively, show the microscopic surfaces with isotropic texture, transverse texture and longitudinal texture.

\subsection{Friction heat and heat flux distribution of sealing structure}

The frictional heat generated by sliding contact between the seal ring and the cylinder causes a sharp increase in the temperature of the contact surface, affecting the overall seal characteristics. The calculating formula for heat flow generated by friction is [23]

$$
Q=c f p_{\mathrm{c}} v S
$$

where $c$ is energy conversion factor and set to $1, f$ is the friction coefficient of contact surface and set to $0.1, p_{c}$ is the contact pressure of sliding contact surface, $v$ is sliding velocity and $S$ is the contact area.

In view of the selected work condition, its temperature rise caused by friction is calculated. According to the 


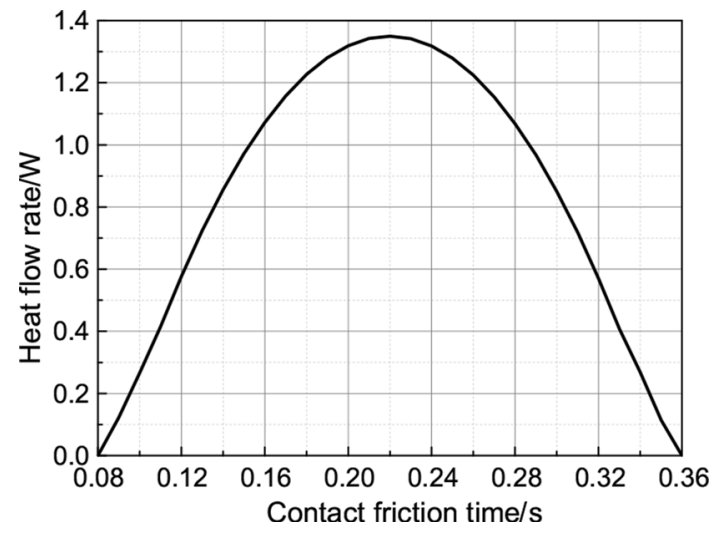

Figure 11. Curve of heat flow rate.

critical velocity obtained in section 2.3 and the design parameter of actuator, the separation time of contact surface of high-speed sliding seal is about $0.36 \mathrm{~s}$. Meanwhile, the sliding velocity increases from 0 to $11 \mathrm{~m} / \mathrm{s}$ and the mean contact pressure of sliding contact surface decreases form 11.05 to $0 \mathrm{MPa}$.

Ouyang et al [24] obtained an approximate linear relation between friction forces of seal ring and sliding velocity, assuming that the relation between mean contact load in calculation domain and the sliding velocity is approximately linear without considering the variation of friction coefficient. The heat flow can be obtained as $Q=-77.62 t^{2}+34.12 t-2.24$. The maximum value is $1.35 \mathrm{~W}$ as shown in figure 11 .

The heat flow needs to be distributed when the parameters mentioned before are determined. As shown in figure 12 , the total heat flow is $Q=Q_{a}+Q_{b}+Q_{c}$, where $Q_{a}$ is the heat flow (W) into A, $Q_{b}$ is the heat flow (W) into B and $Q_{c}$ is the dissipation of heat flow (W).

When the dissipation of heat flow is ignored and the distribution coefficient is introduced, the energy variation of friction pairs can be described quantitatively. According to the theory of thermodynamics, the distribution formula for the friction pairs is as follows:

$$
\delta=\frac{Q_{a}}{Q_{b}}=\sqrt{\frac{K_{a} \rho_{a} C_{a}}{K_{b} \rho_{b} C_{b}}}, \quad Q_{a}=\frac{\delta}{\delta+1} Q
$$

where $K$ is thermal conductivity $\left(\mathrm{W} /\left(\mathrm{m}^{\circ} \mathrm{C}\right)\right), \rho$ is the material density $\left(\mathrm{kg} / \mathrm{m}^{3}\right)$ and $C$ is specific heat capacity of materials $\left(\mathrm{J} / \mathrm{kg}{ }^{\circ} \mathrm{C}\right)$.

The materials of friction pairs include PTFE and steel; the distribution coefficients are $\delta=0.0518$ and $Q_{\alpha \max }=$ $0.07 \mathrm{~W}$. In terms of the inner wall surfaces of cylinder and seal strip, the contact domain changes continuously. In addition, the thermal conductivity of steel is far larger than that of PTFE. Thus, the influence of friction heat on temperature field of metal wall surface could be ignored with the assumption of a constant temperature of $25^{\circ} \mathrm{C}$.

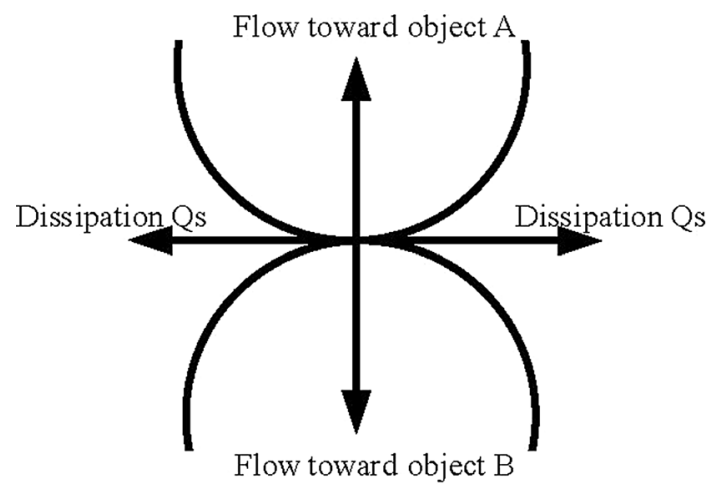

Figure 12. Distribution of heat flow rate.

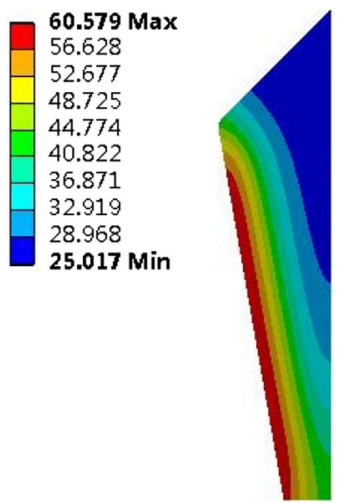

Figure 13. Temperature field of seal ring.

It could be obtained with the working medium state equation that the air temperature in cylinder will rise $70^{\circ} \mathrm{C}$ firstly and then decrease to preliminary temperature. Therefore, when transient temperature field analysis is carried out on the seal ring, the friction heat will be applied to the sliding contact surface in the form of heat flow, and the temperature variation in cylinder is applied to the inner lip surface of $\mathrm{V}$-shaped ring in the form of boundary temperature. The temperature distribution of contact surface could be obtained by ignoring convection heat transfer between seal ring's surface and air.

As the thermal conductivity coefficient of PTFE is very small, the analysis result of only nearby temperature field in the calculation domain is given. It is applied to the fine numerical simulation model for sliding seal lubrication characteristic as the boundary condition. The analysis result of temperature field is shown in figure 13 .

\subsection{Three-dimensional precise numerical model for lubrication performance}

In order to ensure that the width of the computation domain contains enough rough surface features, and considering the computational cost, the size of the computational domain is 


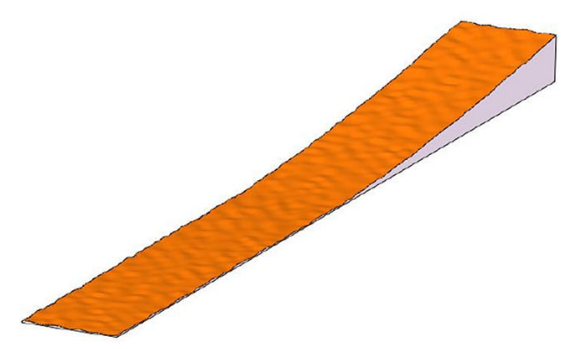

(a) Isotropic texture.

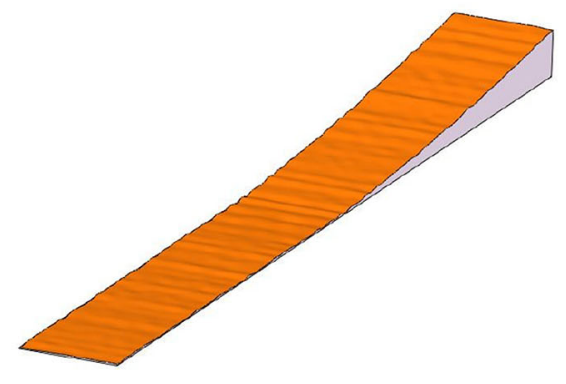

(b) Transverse texture.

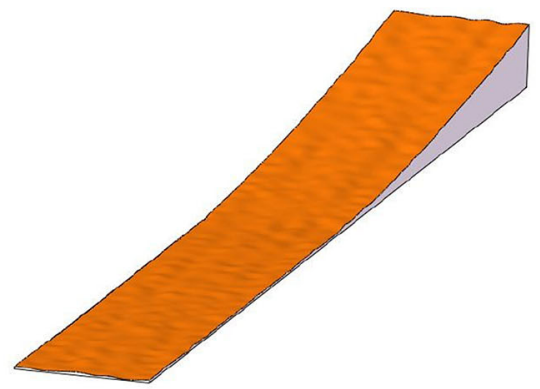

(c) Longitudinal texture.

Figure 14. Calculation domain of three-dimensional oil film.

chosen as $2000 \mu \mathrm{m} \times 200 \mu \mathrm{m}$. The point cloud data are generated form random surface data and the relation equation between $H$ and $X$ in section 2.2. The oil film calculation domain as shown in figure 14 can be generated with the construction method of point cloud.

The flow numerical calculation model in oil film calculation domain is established, and the setting of calculation domain and boundary are shown in figure 15 . The upper surface of calculation domain is the rough surface of seal ring, which is set as wall surface type and is divided into three areas with different assignments to temperature boundaries according to the results of temperature field as shown in figure 13. The left and right surfaces are set as adiabatic wall and the lower surface is dynamic wall surface. The sliding velocity is selected within the nearby domain of obtained critical velocity and the temperature is always $25^{\circ} \mathrm{C}$. The back wall surface is the boundary of pressure entrance and the pressure is $20 \mathrm{MPa}$. The front wall surface is the boundary of pressure existence with a pressure of $0.1 \mathrm{MPa}$. The full cavitation model is selected as the cavitation boundary and the control equation at calculation domain is solved based on SIMPLEC algorithm with finite volume.

The lubrication oil has the density of $800 \mathrm{~kg} / \mathrm{m}^{3}$, the specific heat capacity of $2090 \mathrm{~J} /(\mathrm{kgK})$ and the thermal conductivity of $0.149 \mathrm{w} /(\mathrm{mK})$. The viscosity of lubrication oil could be calculated using the Roelands equation [25]. The viscosity of lubrication oil is embedded in the

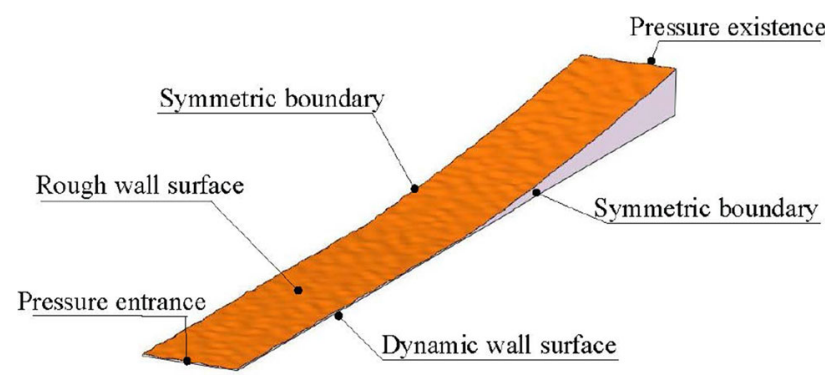

Figure 15. Calculation domain and boundary condition. numerical simulation model with UFD subroutine as follows:

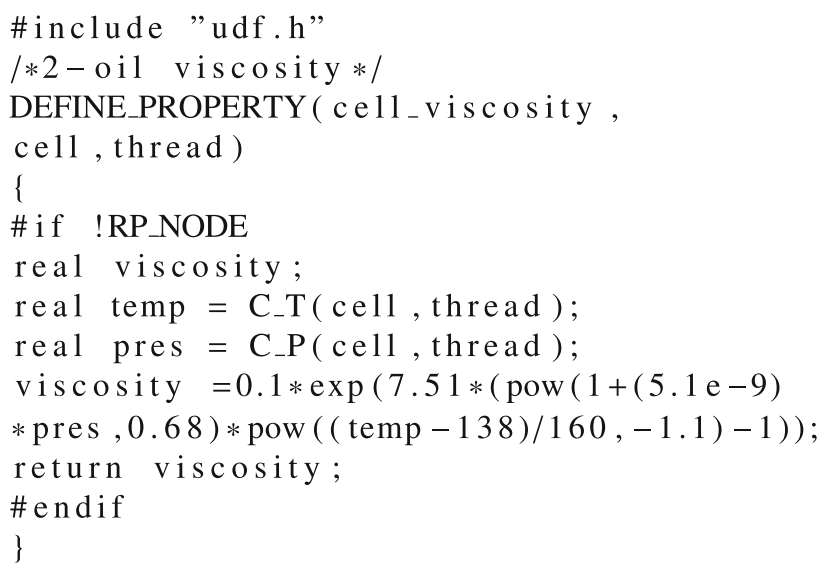

In order to ensure that the numerical results fully reflect the characteristics of rough surface's effects on the dynamic characteristics of oil film, the computational domain is divided into sufficiently small mesh relative to the roughness, and the mesh number is about 8 million.

\subsection{Precise positioning of critical velocity}

The positioning value of critical velocity in seal ring is shown in table 2 , including isotropic microscopic surface as well as microscopic surfaces with transverse texture and longitudinal texture. As indicated, the critical velocity of

Table 2. Critical velocity.

\begin{tabular}{lccc}
\hline Microscopic surface & $\begin{array}{c}\text { Isotropic } \\
\text { texture }\end{array}$ & $\begin{array}{c}\text { Transverse } \\
\text { texture }\end{array}$ & $\begin{array}{c}\text { Longitudinal } \\
\text { texture }\end{array}$ \\
\hline $\begin{array}{l}\text { Precise positioning } \\
\text { value }\end{array}$ & 9.1 & 9.85 & 10.7 \\
$\begin{array}{c}\text { Preliminary } \\
\text { positioning value }\end{array}$ & 11 & & \\
\begin{tabular}{l} 
Error $(\%)$ \\
\hline
\end{tabular} & -17.3 & -10.45 & -2.7 \\
\hline
\end{tabular}




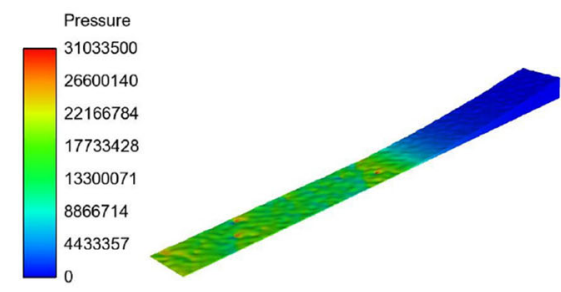

(a) Isotropic texture

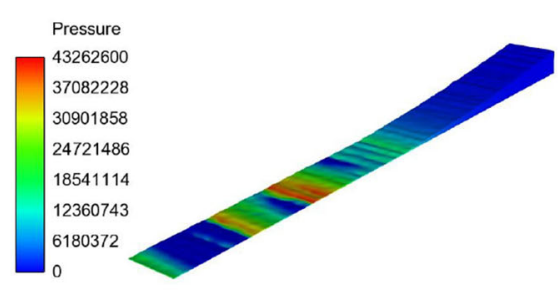

(b) Transverse texture

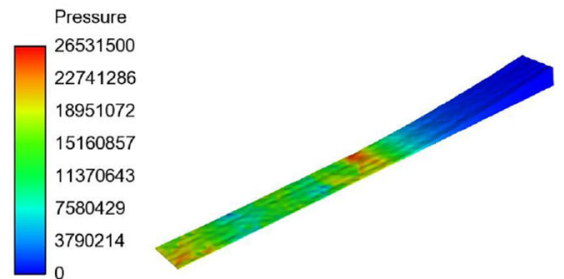

(c) Longitudinal texture

Figure 16. Nephogram of pressure distribution of oil film under critical condition.

isotropic microscopic surface is the lowest while that of the surface with longitudinal texture is the highest.

The nephogram of pressure distribution of oil film with different microscopic surfaces under critical condition is shown in figure 16. As indicated, the oil film pressure distribution with isotropic microscopic surface is the most uniform while that of microscopic surface with transverse texture features is obviously corrugated. In addition, the microscopic surface with longitudinal texture features has the highest oil film pressure concentrated in the transition of convergence domain.

It is concluded that the isotropic microscopic surface has the strongest lubrication ability and the surface with transverse texture is the second, while the microscopic surface with longitudinal texture is the weakest. In general, the lubrication ability of microscopic texture with transverse texture is higher than that of microscopic with longitudinal texture. Also the conclusion is consistent with that obtained by friction experiment in reference [26].

\section{Conclusion}

Targeted on the rodless open cylinder sealing structure, the local structure of sealing structure is improved to enhance its hydrodynamic effect. The following conclusions could be obtained based on the lubrication characteristics analysis of sealing structure:

(1) The preliminary positioning of critical velocity has been achieved by establishing the two-dimensional high-efficiency analysis model, which provides initial computational velocity for further precise positioning of critical velocity. In addition, the precise positioning of critical velocity could be achieved in its nearby area. Due to significant reduction of the velocity range for the calculation of critical velocity's precise positioning, the calculation method for critical velocity could improve the solution efficiency significantly, which combines the two-dimensional high-efficiency analysis model with three-dimensional precise numerical analysis model.

(2) On the premise of satisfying the seal requirement, smaller front lip angle and pre-compression should be adopted. Under the condition of low temperature, the lubrication oil with smaller viscosity should be used to form the lubricant film as soon as possible. Under the condition of high temperature, the lubrication oil with larger viscosity should be used to improve the carrying capacity of lubricant film.

(3) Combined with Gauss distribution function and point cloud method, the three-dimensional precise numerical analysis model is established. Thus the precise positioning of critical velocity has been achieved and the influence mechanism of texture feature on lubrication performance has been further revealed. Later, the conclusion of easier formation of isotropic microscopic surface was obtained. The research results provide theoretical support for the design of the sealing structure and the optimization of lubrication performance in the rodless open cylinder.

\section{Acknowledgements}

Grateful thanks for the financial supports from Innovation Partnership Fund for Universities of China Aerospace Science and Technology Corporation (CASC), Natural Science Funds for Young Scholar of Jiangsu Province (No. BK20170-837) and the fundamental research funds for the central universities (No. 309181B8807). My thanks also go to the experts and the editors for their work.

\section{References}

[1] Morimoto T, Aliff M, Akagi T, et al 2016 Development of flexible haptic robot arm using flexible pneumatic cylinders with backdrivability for bilateral control. In: Proceedings of the 3rd International Conference on Intelligent Technologies and Engineering Systems (ICITES2014), Lecture 345, pp. 231-237

[2] Wu T C 2015 U.S. Patent No. 9,027,461. Washington, DC: U.S. Patent and Trademark Office.

[3] Rana A S and Sayles R S 2005 An experimental study on the friction behaviour of aircraft hydraulic actuator elastomeric reciprocating seals. In: Tribology and Interface Engineering, vol. 48 , pp. 507-515 
[4] Bhaumik S, Kumaraswamy A and Guruprasad S 2013 Design and development of test rig for investigation of contact mechanics phenomena in reciprocating hydraulic seals. Procedia Engineering 64: 835-843

[5] Nikas G K, Almond R V and Burridge G 2014 Experimental study of leakage and friction of rectangular, elastomeric hydraulic seals for reciprocating motion from -54 to $+135^{\circ} \mathrm{C}$ and pressures from 3.4 to 34.5 MPa. Tribology Transactions 57: 846-865

[6] Salant R F, Maser N and Yang B 2007 Numerical model of a reciprocating hydraulic rod seal. Journal of Tribology 129(1): 91-97

[7] Thatte A and Salant R F 2009 Transient EHL analysis of an elastomeric hydraulic seal. Tribology International 42(10): 1424-1432

[8] Yang B and Salant R F 2009 Numerical analysis compares the lubrication of U seal and step seal. Sealing Technology 3: 7-11

[9] Yang B, Salant R and Woodruff G W 2011 Simulation of O-ring and U-cup seals for a mini-actuator. Sealing Technology 11: 8-12

[10] Schmidt T, André M and Poll G A 2010 Transient 2D-finiteelement approach for the simulation of mixed lubrication effects of reciprocating hydraulic rod seals. Tribology International 43: 1775-1785

[11] Crudu M, Fatu A, Hajjam M and Cristescu C 2013 Numerical and experimental study of reciprocating rod seals including surface roughness effects. Sealing Technology 6: 8-11

[12] Huang Y 2014 Elastohydrodynamic model of hydraulic rod seals with various rod surfaces. Doctoral Dissertation, Georgia Institute of Technology

[13] Huang Y and Salant R F 2015 Numerical analysis of a hydraulic rod seal: flooded vs. starved conditions. Tribology International 92: 577-584

[14] Huang Y and Salant R F 2016 Simulation of a hydraulic rod seal with a textured rod and starvation. Tribology International 95: 306-315
[15] Ouyang X P, Xue Z, Peng C, et al 2015 Performance analysis on VL seal in aircraft cylinder. Journal of Zhejiang University 49: 1755-1761

[16] Cheng D X 2010 Mechanical design manual, 5th ed. Beijing: Machinery Industry Press

[17] Xiao-liu Y 2013 Tribology and lubrication technology. Hefei: HeFei University of Technology Press

[18] Hui-fang X, Quan Y, Yi-min S, et al 2016 Dynamic friction characteristics of sliding rough interfaces in line contact under labrication. Journal of Vibration and Shock 35: 188-194

[19] Shi-zhu W and Ping H 2008 Tribology principle, 3rd ed. Beijing: Tsinghua University Press

[20] Gero L R and Ettles C M McC 1986 An evaluation of finite difference and finite element methods for the solution of the Reynolds equation. ASLE Transactions 29: 166-172

[21] Zhong J L, Ren J, Da-Wei M A, et al 2015 Engineering Mechanics 32: 229-235

[22] Xiang-kui L, Wen-jian Y, Guo-liang X, et al 2015 The influence of characteristic of rough on gas sealing performance in sealing structure. Journal of Mechanical Engineering 51: 110-115

[23] Gecim B and Winer W O 1985 Transient temperatures in the vicinity of an asperity contact. Journal of Tribology 107: 333-341

[24] Ouyang X P, Xue Z Q, Peng C, et al 2016 Analysis on aircraft cylinder seal property based on mixed lubrication theory. Journal of Beijing University of Aeronautics and Astronautics 42: 251-257

[25] Ping H 2011 Numerical calculation method of lubrication. Beijing: Higher Education Press

[26] Wang S, Hu Y Z, Wang W Z, et al 2007 Effects of surface roughness on sliding friction in lubricated-point contacts: experimental and numerical studies. Journal of Tribology 129: 809-817 